\title{
Progress on electron cloud effects calculations for the FNAL main injector*
}

\author{
Kiran G. Sonnad, ${ }^{\dagger}$ Miguel A. Furman, and Jean-Luc Vay \\ Center for Beam Physics \\ Lawrence Berkeley National Laboratory, \\ Berkeley, CA 94720-8211
}

(Dated: 9 June 2008)

\begin{abstract}
We have studied the response of the beam to an electron cloud for the Fermilab Main Injector using the Quasistatic Model [1] implemented into the particle-in-cell code Warp [2]. Specifically, we have addressed the effects due to varying the beam intensity, electron cloud density and chromaticity. In addition, we have estimated the contribution to emittance evolution due to beam space-charge effects. We have carried out a comparison between how the beam responds at injection energy and at top energy. We also present some results on the validation of the computational model, and report on progress towards improving the computational model.
\end{abstract}

\section{INTRODUCTION}

Electron-cloud-induced instabilities are caused by an incoherent tune shift created by the variation in focusing along the bunch and also the nonlinear spacecharge forces caused by the electrons. Modeling this phenomenon is complex because it involves tracking of the beam particles and electrons, along with calculation of their respective space-charge forces. We have made a systematic study of this computation based on the parameters of the Main Injector (MI) and also on the results obtained from the build-up calculation using the code POSINST [3]. The results show a general dilution of emittance with increased electron density and increased beam intensity. Although these values are correlated, it is important to study their individual effects for a better understanding of the dynamics. The model adopted here assumes an initially uniform electron distribution. When the beam encounters these electrons, they move closer toward the beam leading to a pinching effect. As a result, the tail of the bunch will encounter a more dense electron distribution and thereby a higher tune shift compared to the head. This interaction also creates a nonuniform distribution of electrons leading to a nonlinear focusing effect.

\section{A BRIEF DISCUSSION ON THE MODEL}

Warp [2] is a simulation program that has been developed at LLNL and LBNL for studying phenomena in heavy ion fusion experiments. This is a 3-D electrostatic particle-in-cell (PIC) code that can also run in parallel on multiple processors. Considerable progress has been made in implementing the quasistatic model [1] in Warp

\footnotetext{
*Work supported by the FNAL Main Injector upgrade effort and by the US DOE under contract no. DE-AC02-05CH11231.

${ }^{\dagger}$ Now at Institute for Synchrotron Radiation, Forschungszentrum Karlsruhe, P. O. Box 3640, D-76021 Karlsruhe, Germany; Electronic address: Kiran.Sonnad@iss.fzk.de
}

to study electron cloud effects on the beam in circular accelerators [4] . The quasistatic-mode in Warp has been developed with a scheme similar to that in HEADTAIL [5] developed at CERN, QUICKPIC [6] developed at UCLA, and CMAD at SLAC [7]. The scheme involves modeling the beam space charge in the form of a series of slices, each of whose charge distribution is deposited onto a series of corresponding two dimensional grids. On the other hand, the electron cloud distribution is deposited on to a single two dimensional grid. The beam is made to pass through the electron cloud slice by slice and the charge distributions of the two species are evolved accordingly. This set of interactions can be chosen at any number of points in the storage ring which are referred to here as "stations." The evolution of the beam macro particles between two adjacent stations is determined by a single transfer map which follows the transverse betatron motion and longitudinal synchrotron motion. The effect of the beam space-charge and of the electrons on the beam is modeled as velocity kicks applied at each station. Thus, the single particle transfer map is concatenated with the series of "kicks" along the length of the bunch occurring due to the presence of the electron cloud. Results from this code have been benchmarked against those obtained by HEADTAIL for parameters representing that of LHC. Details of this effort are provided later in this report. A schematic of the simulation model is shown in Figs. 1 and 2.

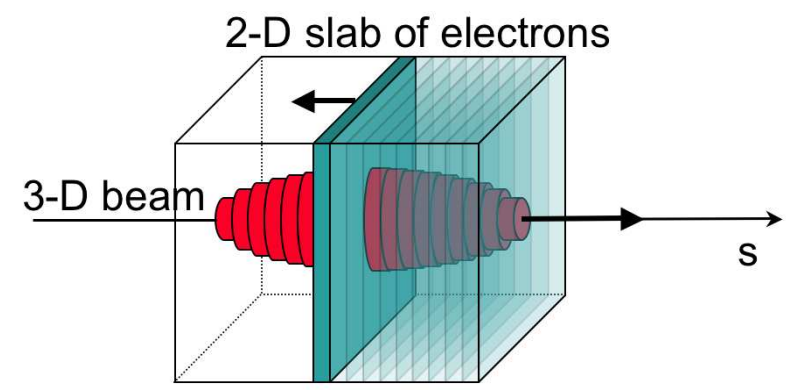

FIG. 1: A schematic of the model used for the electron-beam interaction calculations 


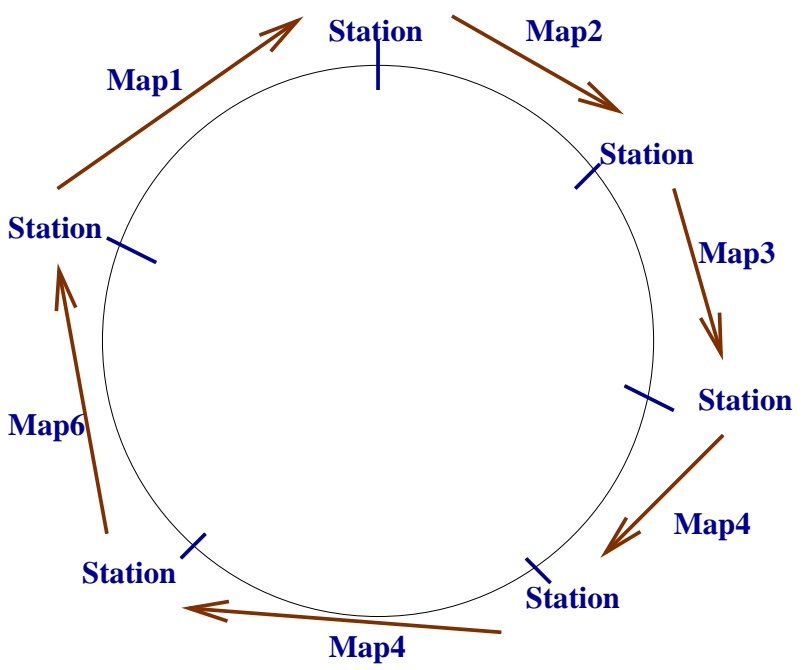

FIG. 2: A schematic of the model used for particle tracking

TABLE I: Machine parameters used in the simulations

\begin{tabular}{lr}
\hline \hline Beam injection energy & $8.9 \mathrm{GeV}$ \\
Beam top energy & $120 \mathrm{GeV}$ \\
Initial $x$ and $y$ rms emittance at injection & $0.26 \mathrm{~mm}-\mathrm{mr}$ \\
Initial $x$ and $y$ rms emittance at top energy & $0.117 \mathrm{~mm}-\mathrm{mr}$ \\
rms bunch length at injection & $0.75 \mathrm{~m}$ \\
rms bunch length at top energy & $0.15 \mathrm{~m}$ \\
Horizontal, vertical tune & $26.424996,25.415003$ \\
Synchrotron tune at injection & 0.0051712 \\
Synchrotron tune at top energy & 0.0014082 \\
Average $\beta_{x}, \beta_{y}$ & $19.992,20.7868 \mathrm{~m}$ \\
Chamber cross section & elliptical \\
Horiz., vert. chamber size & $12.3,4.9 \mathrm{~cm}$ \\
\hline \hline
\end{tabular}

\section{COMPUTATIONAL AND MACHINE PARAMETERS}

The machine parameters represent the current working conditions and lattice of the MI. For the purposes of this Section, we assume a uniform focusing model, in which the average beta functions were calculated from the tune and the circumference of the MI. The focusing force was applied via transfer maps obtained from the average beta functions, assumming a constant phase advance between the "stations." The complete set of parameters used in calculations is given in Table I.

The computational parameters are indicated in Tab. II. In order to optimize between computation time and calculational accuracy, before deciding upon this parameter set, we carried out a few runs with more refined parameters, such as more stations (up to 100), a finer grid $(128 \times 128)$, a larger number of macroparticles, etc. In a separate Section below we report on our progress made in implementing a FODO cell optics in the calculation instead of the continuous focusing scheme. As will be indicated later, the continuous focusing model in Warp shows good agreement with HEADTAIL results.
TABLE II: Computational parameters

\begin{tabular}{lr}
\hline \hline Grid & $64 \times 64$ \\
Number of macro protons & 60000 \\
Number of beam slices & 70 \\
Number of macro electrons & 4096 \\
Number of stations & 10 \\
Initial beam offset in $x, y$ & $\sqrt{0.1} \sigma_{x}, \sqrt{0.1} \sigma_{y}$ \\
\hline \hline
\end{tabular}

\section{DEPENDENCE ON BEAM INTENSITY}

Increasing the beam intensity leads to a greater spacecharge force thereby attracting more electrons toward the beam. This leads to a tune increase, affecting the phase and magnitude of the coupled oscillations of the beam and electrons centroids about each other. The increased pinching effect leads to a greater variation of tune along the bunch. In this study we used a constant electron density $n_{e}=1 \times 10^{12} \mathrm{~m}^{-3}$ and bunch intensities $N_{p}=$ $6 \times 10^{10}, 1.1 \times 10^{11}, 2 \times 10^{11}$ and $3 \times 10^{11}$. All other parameters corresponded to injection energy conditions.

Figure 3 clearly shows that increasing the beam intensity leads to increased emittance dilution. The vertical emittance growth is higher, the reason for which is un-

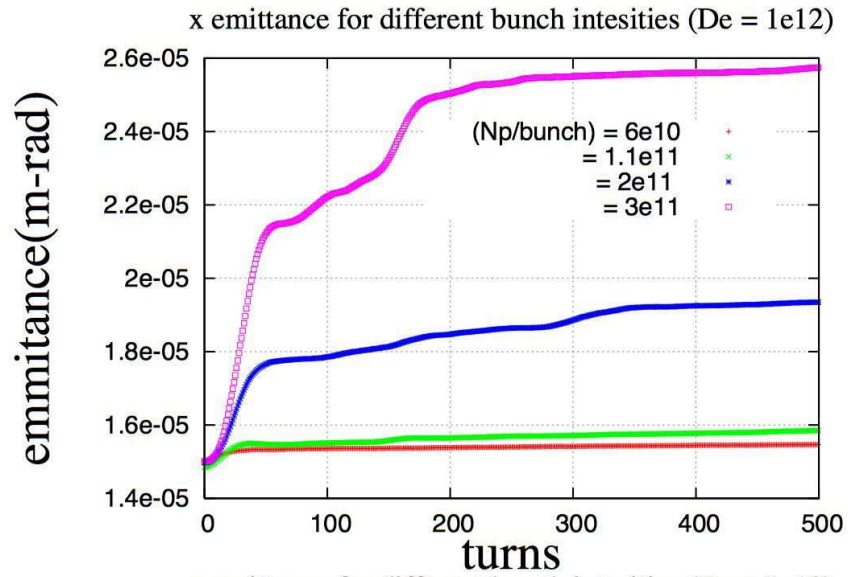

y emittance for different bunch intesities $(\mathrm{De}=1 \mathrm{e} 12)$

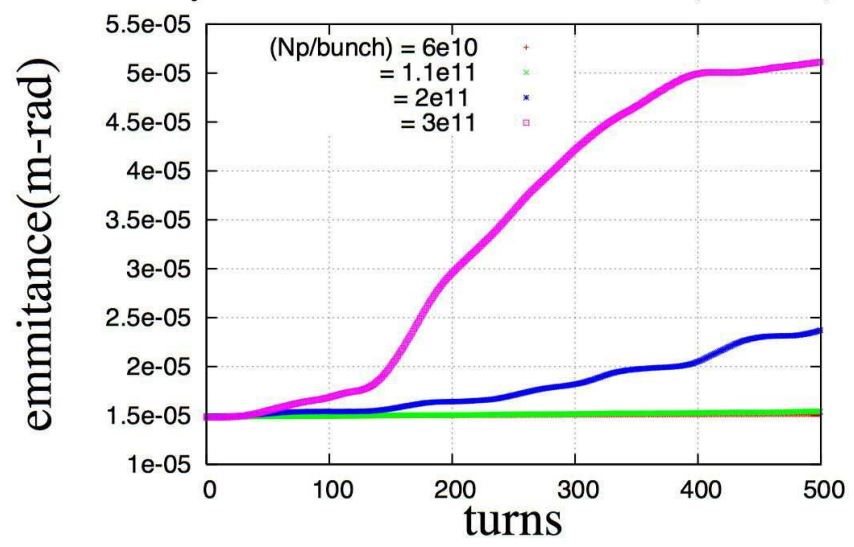

FIG. 3: Evolution of the beam emittances for varying beam intensities 
clear at this point. This is an interesting result given that the initial beam emittances are the same in both planes, and the values of the respective beta functions are almost equal hence the initial beam sizes are almost the same in the two planes. It is likely that the small difference in the horizontal and vertical tunes, or the asymmetry of the vacuum chamber, is the cause of this (in this study we neglect the curvature of the actual machine orbit, so the simulated beam travels in a straight line).

\section{EFFECT OF ELECTRON DENSITY}

In this study, we keep the bunch population $N_{p}$ constant at $3 \times 10^{11}$, and vary the electron density $n_{e}$. A higher electron density leads to a higher tune shift and tune spread. One could expect the electron cloud spacecharge forces to become more nonlinear with increased $n_{e}$. An increased $n_{e}$, in turn, directly increases the headtail interaction in the bunch. The electron densities used here are $n_{e}=1 \times 10^{10}, 1 \times 10^{11}, 1 \times 10^{12}, 5 \times 10^{12}$ and $1 \times 10^{13} \mathrm{~m}^{-3}$. The results are shown in Fig. 4 We still see a larger emittance growth along the vertical direction than in the horizontal. We also see a higher emittance growth for an electron density of $1 \times 10^{12}$ than $5 \times 10^{12}$ in the vertical plane. This could be attributed to an unfavorable coupling occurring between the $x$ and $y$ motion at $n_{e}=1 \times 10^{12} \mathrm{~m}^{-3}$.

\section{SIMULATION RESULTS BASED ON ELECTRON DENSITY ESTIMATES OBTAINED FROM POSINST}

In this section, we show results obtained using values for corresponding to a given bunch intensity. The electron density $n_{e}$ was first obtained with the electron-cloud build-up code POSINST, and then used as an input to Warp to compute the emittance growth for the corresponding value of the bunch intensity $N_{p}$. The details of these electron-cloud build-up calculations can be found in $[3]$.

Table III provides the values of $n_{e}$ for each value of $N_{p}$ obtained with POSINST, assuming a peak secondary emission yield (SEY) of 1.3. This value of the SEY is believed to be a reasonable estimate of the actual SEY of the chamber surface in the conditioning state in mid2007 [8]. The second column is the electron density averaged within one beam sigma, and the third column is the density averaged over the whole chamber. The electron densities we used as input in the Warp calculations were the mean of these two numbers for each corresponding value of $N_{p}$.

The calculations of the emittance growth using these values are shown in Fig. 5 . It is clear that the combined effect of increasing $N_{p}$ and increased $n_{e}$ degrades the emittance considerably. The calculations show that the current working conditions of the MI, with $N_{p}=6 \times 10^{10}$,
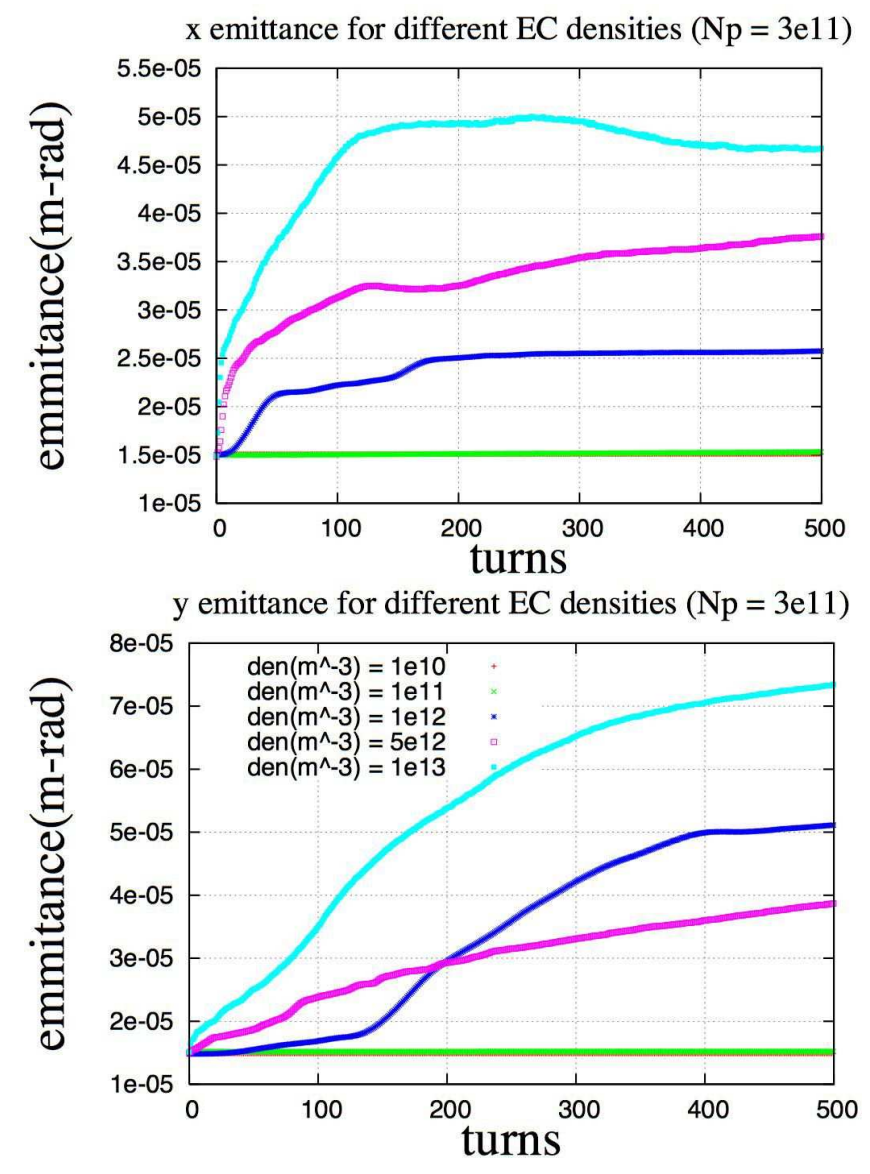

FIG. 4: Evolution of beam emittances for varying electron densities

TABLE III: Bunch intensity and corresponding electron density $\left(\mathrm{m}^{-3}\right)$ obtained from POSINST

\begin{tabular}{lrr}
\hline \hline$N_{p}$ & ave. $n_{e}$-beam sigma & ave. $n_{e^{- \text {chamber }}}$ \\
\hline $6.0 \times 10^{10}$ & $2.81 \times 10^{7}$ & $1.30 \times 10^{7}$ \\
$1.0 \times 10^{11}$ & $1.33 \times 10^{8}$ & $6.42 \times 10^{7}$ \\
$1.5 \times 10^{11}$ & $2.08 \times 10^{10}$ & $1.05 \times 10^{10}$ \\
$2.0 \times 10^{11}$ & $3.72 \times 10^{12}$ & $1.98 \times 10^{12}$ \\
$3.0 \times 10^{11}$ & $1.20 \times 10^{13}$ & $6.86 \times 10^{12}$ \\
\hline \hline
\end{tabular}

do not pose a problem. The maximum achievable intensity at present, $N_{p} \simeq 1.1 \times 10^{11}$, does not lead to a noticeable emittance growth either. However, for higher values of $N_{p}$, and correspondingly higher values of $n_{e}$, considerable emittance growth is predicted.

\section{BEAM SPACE-CHARGE EFFECTS}

The beam space-charge-induced tune shift at injection has been estimated to be $\sim 0.3$ at $N_{p}=3 \times 10^{11}$. Thus, it is important to perform a study to understand the combined effect of electron cloud and beam space-charge forces. It may be noted that these forces act in opposite 


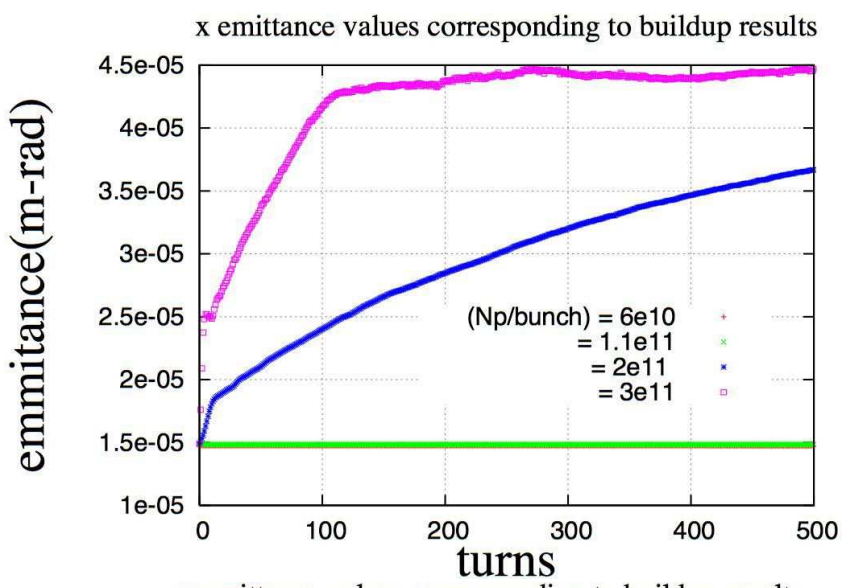

y emittance values corresponding to buildup results

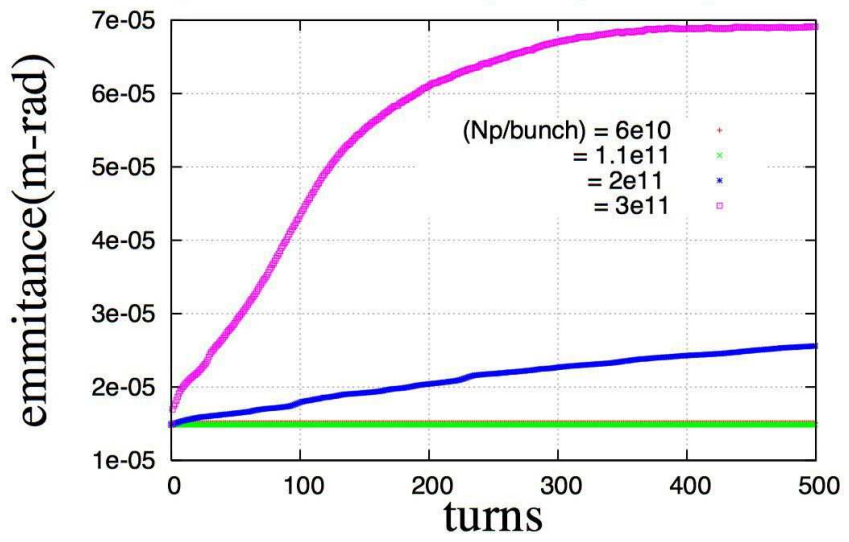

FIG. 5: Evolution of the beam emittances using values obtainted from POSINST

directions, and yet, adding these forces is expected to lead to greater degradation in emittance because both the forces are nonlinear and their combination need not lead to cancellation.

Figures 6 show the emittance growth for different beam intensities. When compared with Figs. 3 we see that the effect of space charge leads to greater emittance growth than the electron cloud.

\section{ELECTRON CLOUD EFFECT AT TOP BEAM ENERGY}

At higher energies, we have two competing effects taking place. The beam becomes stiffer, due to which the distribution would be more stable causing less emittance growth. On the other hand, the transverse and longitudinal size of the beam is considerably reduced, as a result of which, the electrons feel a higher field in the vicinity of the beam. This could cause more electrons to converge toward the beam during a bunch passage due to which, the beam would experience a higher head-tail interaction caused by the progressive pinching of electrons with bunch passage. Besides, the phase and amplitude of the transverse coupled oscillations between the beam and

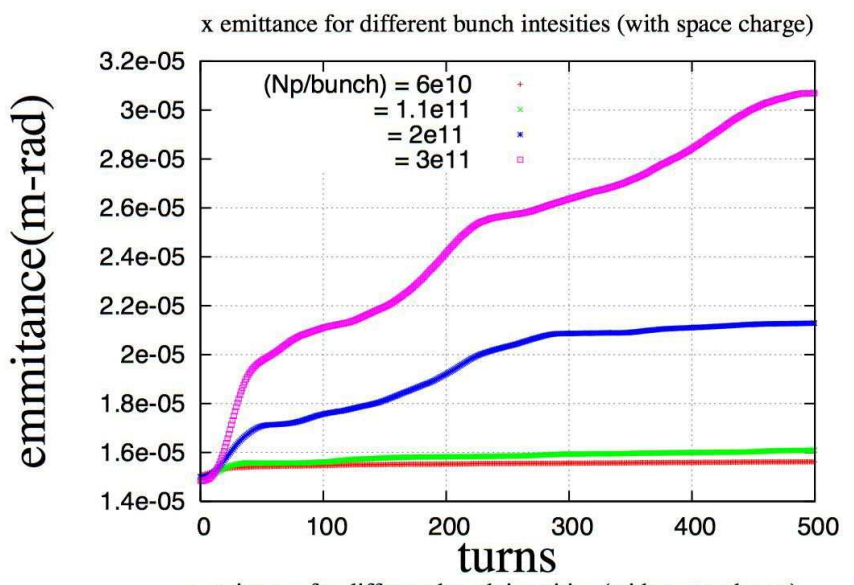

y emittance for different bunch intesities (with space charge)

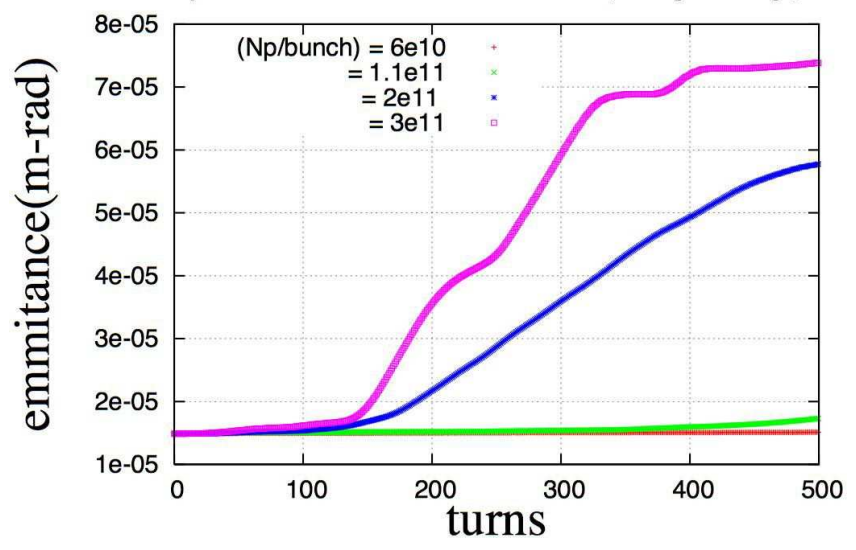

FIG. 6: Evolution of the beam emittances with beam space charge effects included.

the electrons centroid will be different at higher energy. Whether they result in more or less emittance growth is to be determined. Thus, electron cloud effects at higher energies cannot be discounted.

Figure 7 shows the emittance evolution for different beam intensities. The initial emittance, bunch length and synchrotron tunes were changed according to values given in Table I. The chosen electron density was $n_{e}=5 \times 10^{13}$. This rather high value was chosen purely to understand if the beam stiffness would be large enough to control electron effects. The results clearly show that this is not the case. More study needs to be done in this parameter regime.

\section{EFFECT OF CHROMATICITY}

It is well known that chromaticity $\eta$ has a stabilizing effect over various collective effects. This is particularly true in the headtail interaction [9]. Thus, it is important to study the effect of varying $\eta$ on the beam evolution with respect to electron cloud effects.

Figure 8 shows the evolution of emittance for different positive and negative values of $\eta$ (we define $\eta$ to be normalized to the tune, that is $\eta=(\partial Q / \partial \delta) / Q$, with 

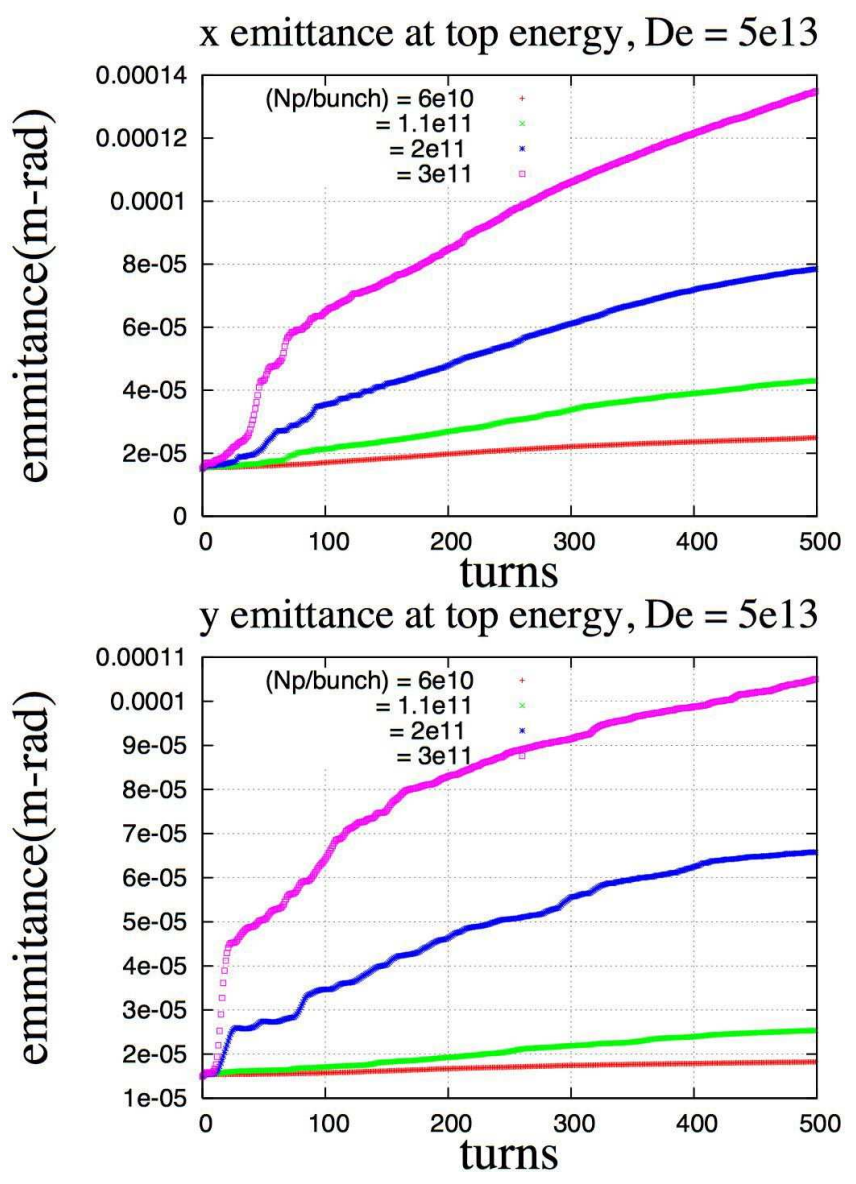

FIG. 7: Evolution of the beam emittances at top energy.

$\delta=\Delta E / E)$. The bunch intensity used in the calculation was $N_{p}=3 \times 10^{11}$ and the electron density $n_{e}=10^{12}$ $\mathrm{m}^{-3}$. The plots indicate that a horizontal chromaticity $\eta_{x}=-0.02543$ and vertical chromaticity $\eta_{y}=-0.02589$ resulted in a minimum emittance growth. The negative value of the optimum $\eta$ is possibly related to the fact that the MI operates below transition at injection. Figure 9 shows the final emittance after 500 turns for different values of $\eta$. These results show that a more detailed study of dependence of electron clouds effects on $\eta$ would be useful when the MI reaches beam intensities for which electron cloud effects become important.

\section{COMPARISONS WITH OTHER CODES}

We have been actively interacting with colleagues at CERN and at SLAC to validate results from Warp against other independent calculations. In this regard, parameters used so far represent the LHC and in some instances, extreme conditions, such as a very high electron density, high synchrotron tune, high chromaticity etc., were used for the sole purposes of comparison of results. The results in Fig. 10 indicate very good agreement with calculations using the code HEADTAIL [5].
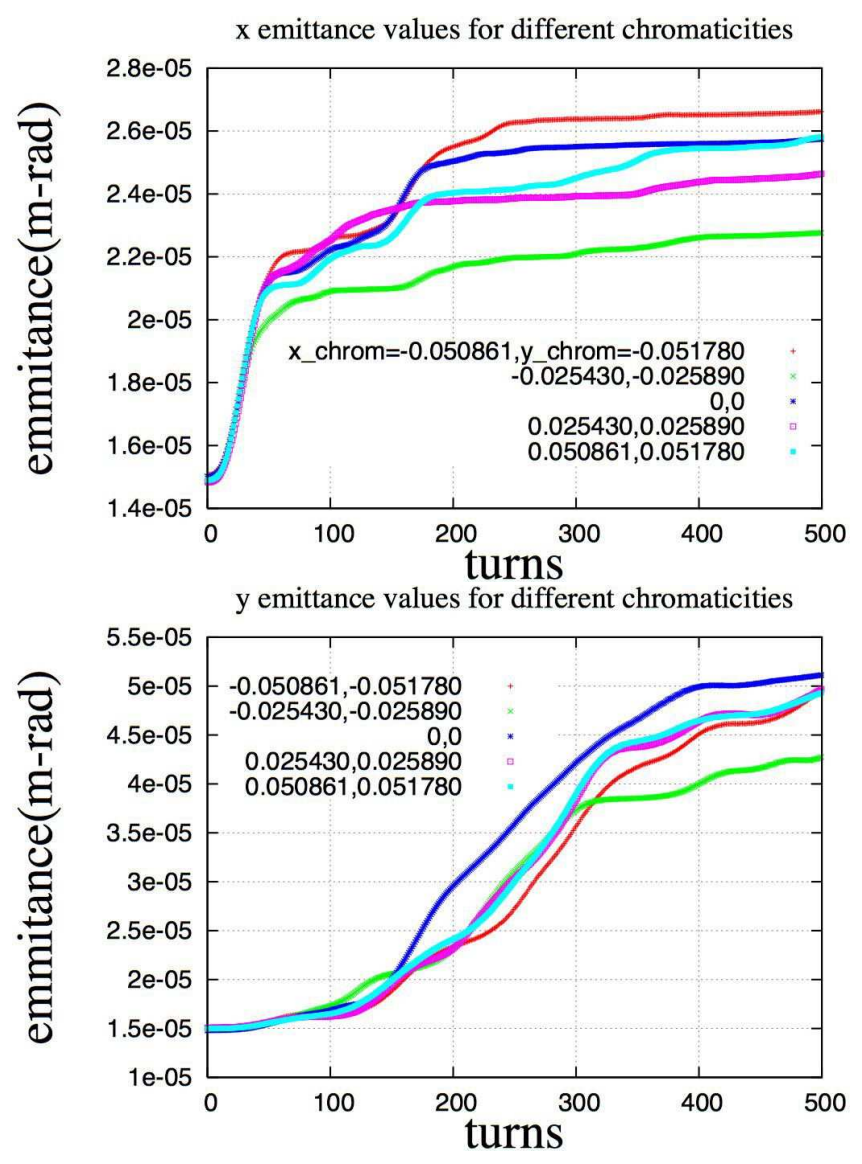

FIG. 8: Evolution of horizontal beam emittance with chromaticities.

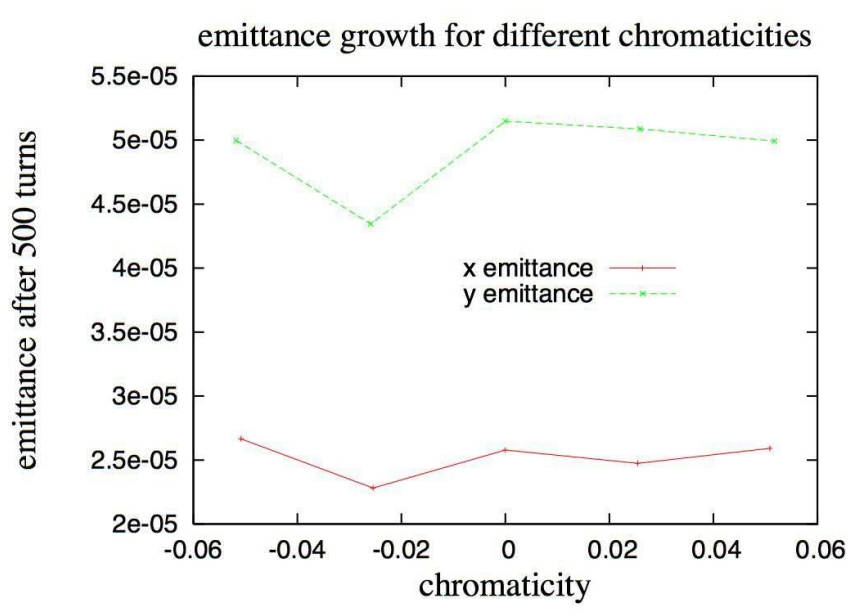

FIG. 9: Final emittance after 500 turns.

Progress on code comparisions between HEADTAIL and Warp has been documented; details are updated on the CERN website dedicated to this effort [10]. 

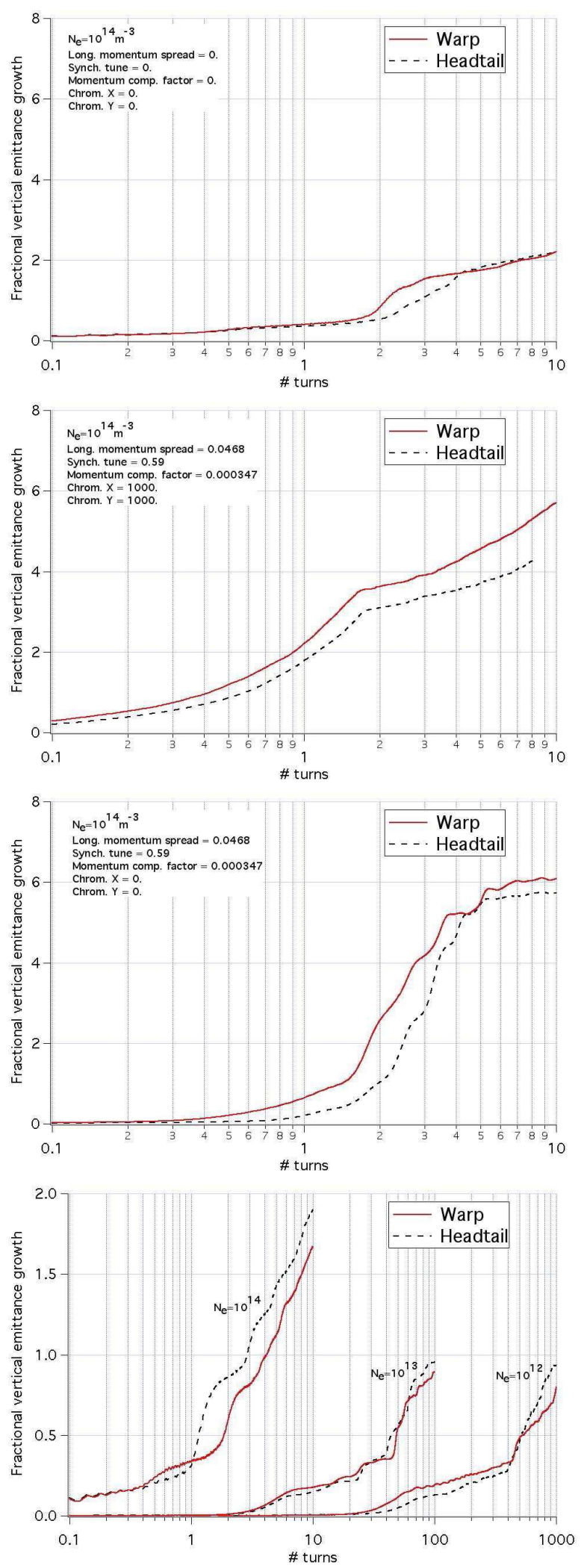

FIG. 10: Comparison of results obtained from Warp and Headtail

\section{PROGRESS ON TRACKING WITH A LATTICE}

We have made significant progress in tracking the beam through actual lattice optics instead of using the smooth focusing model. There is an ongoing effort to compare results between the codes CMAD [7] and HEADTAIL [5]. The scheme involves implementing a FODO lattice that has the same periodicity as that of the machine. However, the strengths of the quads are adjusted such that the tunes produced by this FODO structure match the actual tunes of the machine. The FODO structure can involve quads, drifts and bends. This model has been successfully implemented in Warp and we have compared results with those obtained using CMAD for tracking in the absence of electron clouds. Efforts are under way for making further comparisons using a variety of electron cloud densities. Currently, the SPS parameters are being used for the purpose of this benchmarking effort. Once we complete our calculations and comparisons with other codes using the FODO scheme, we will be in a position to perform calculations for the MI parameters.

\section{DAMPING OF OSCILLATIONS ALONG THE SMALLER DIMENSION OF AN ELLIPTICAL CHAMBER FOR HIGH $N_{p}$ AND $n_{e}$}

We observed that the oscillation of the centroid of the simulated beam was strongly damped along the smaller dimension of the elliptical chamber. All simulations were begun with an initial beam centroid offset $(\Delta x, \Delta y)=$ $\left(\sqrt{0.1} \sigma_{x}, \sqrt{0.1} \sigma_{y}\right)$; beam space-charge effects were not included in this exercise. Two set of parameters were used, which were (a) $N_{p}=6 \times 10^{10}$ with a corresponding $n_{e}=2 \times 10^{7} \mathrm{~m}^{-3}$, and (b) $N_{p}=3 \times 10^{11}$ with $n_{e}=6 \times 10^{12} \mathrm{~m}^{-3}$. Figure 11 clearly indicates that the motion along $y$ is strongly damped for case (b).

It was at first unclear if this damping was a result of the shape of the vacuum chamber or the small difference in the tunes. We then rotated the orientation of the ellipse so that the larger dimension was along $y$ instead of $x$, keeping all other parameters unchanged. Results of tracking the beam centroid clearly showed that the motion in $x$ was then damped for case (b). In addition, when we simulated a round chamber, there was no preferred centroid damping direction, clearly indicating that the effect is due to the motion along the smaller dimension and not to the difference in the tunes. We do not know at present what fraction of the energy contained in the coherent centroid motion went into heating the electron cloud or increasing the emittance growth. These results are preliminary need further investigation and explanation before any firm conclusion can be drawn. 

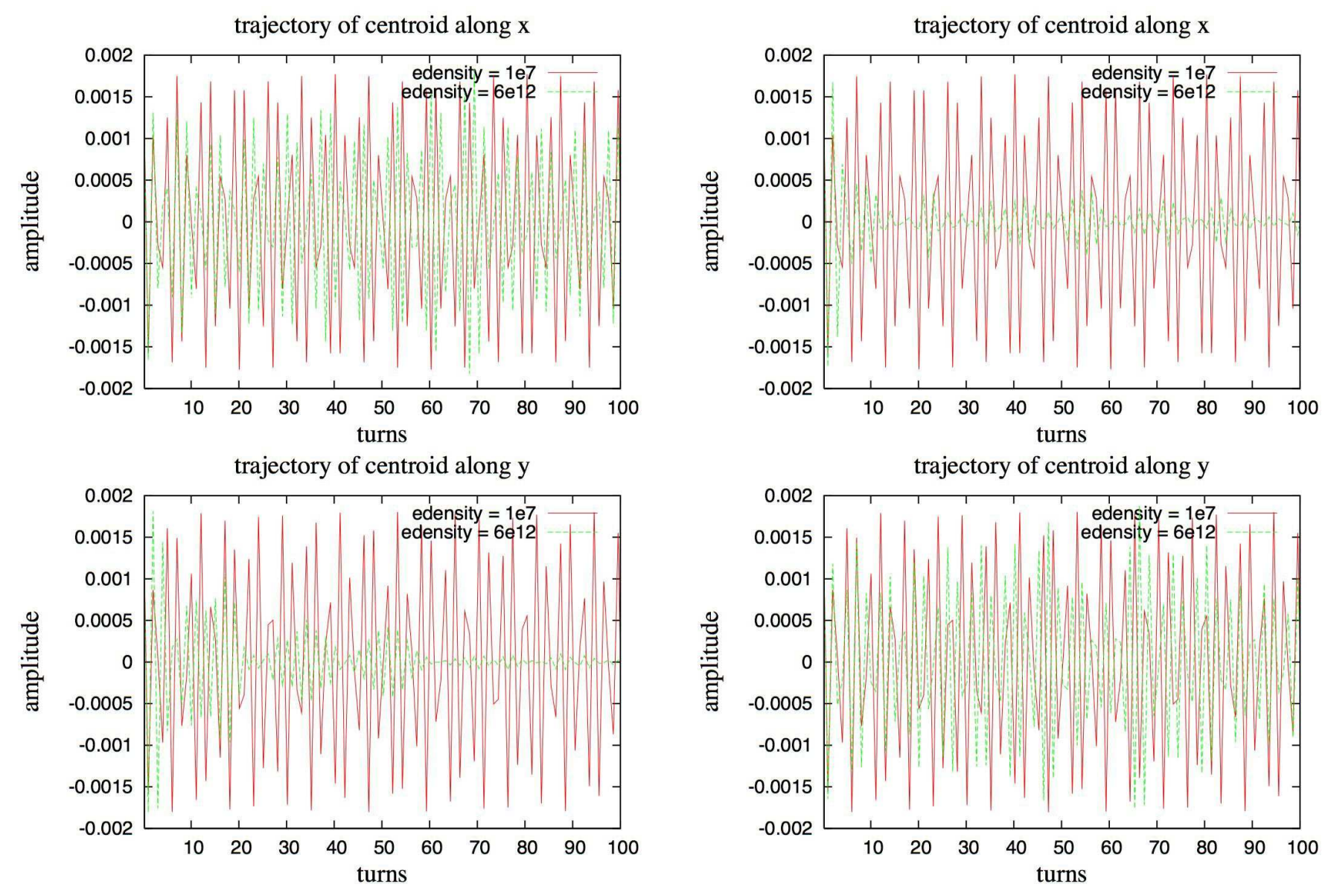

FIG. 11: Damping of beam centroid oscillations.

FIG. 12: Damping of beam centroid oscillations with the chamber artificially rotated by $90^{\circ}$.

\section{CONCLUSION}

In this report we presented results from a detailed study for the present working conditions and possible upgrade parameters for the Fermilab MI. The results show that beam emittance increases significantly with increasing beam intensity $N_{p}$ and increasing electron cloud density $n_{e}$. In a first exercise, we varied $N_{p}$ and $n_{e}$ independently. However, $n_{e}$ depends strongly upon $N_{p}$. Therefore, in a second exercise, we used as inputs to the simulation the values of $n_{e}$ corresponding to a few selected values of $N_{p}$, obtained with the code POSINST, and then studied the emittance evolution. Our results show no significant emittance growth under present working conditions $\left(N_{p}=6 \times 10^{10}\right)$, nor for the maximum presently achievable bunch intensity $\left(N_{p} \simeq 1.1 \times 10^{11}\right)$. However, a significant emittance growth is predicted for higher values of $N_{p}$ and $n_{e}$.

Calculations showed that the effect of beam space charge is considerable at injection, although we expect this to become less significant at higher energies. We also showed that electron cloud effects need not be negligible at the top energy of the MI. Parameter space needs to be studied in more detail with respect to electron build-up

to obtain the correct electron densities; in the present study we have used a rather high value of $n_{e}$ for the purposes of obtaining initial results. We have quantified the effect of chromaticity on emittance growth. Further studies can be done on chromaticities by taking into account what values can be tolerated by the MI lattice without suffering a loss in acceptance.

Considerable progress has been made in validating the Warp-Quasistatic calculations through a comparison with independent calculations made by HEADTAIL. Progress on adding a lattice in the particle tracking of the beam has been reported, and efforts in benchmarking these results with other codes is currenly in progress.

In conclusion, the results obtained so far indicate that electron cloud effects need to be studied in further detail to understand the limitations it could introduce in the upgrade goals of the MI. Finally, the recent result indicating damping of the beam centroid motion along the smaller dimension of the elliptical chamber needs to be investigated further. 


\section{Acknowledgments}

We are grateful to C. Celata and M. Venturini for many discussions, to D. Grote for support with the code
Warp, to E. Benedetto, M. Pivi, G. Rumolo, R. Tomás and F. Zimmermann for assistance in carrying out the code benchmarking exercise, and to I. Kourbanis and R. Zwaska for information on the MI.
[1] P. Sprangle, E. Esarey and A. Ting, Phys. Rev. Lett. 64, 2011-2014 (1990).

[2] D. P. Grote, A. Friedman, J.-L. Vay and I. Haber, AIP Conf. Proc. 55, 749 (2005).

[3] M. A. Furman and G. R. Lambertson, Proc. Intl. Workshop on Multibunch Instabilities in Future Electron and Positron Accelerators "MBI-97" (KEK, Tsukuba, Japan, 15-18 July 1997, KEK Proceedings 97-17, Dec. 1997; Y. H. Chin, ed.), p. 170. M. A. Furman, LBNL-41482/CBP Note 247/LHC Project Report 180, May 20, 1998. M. A. Furman and M. T. F. Pivi, PRST-AB 5, 124404 (2003).

[4] J.-L. Vay, A. Friedman and D. P. Grote, Proc. 9th ICAP 1, 262 (2006).

[5] G. Rumolo and F. Zimmermann, PRST-AB 5, 121002 (2002).

[6] G. Rumolo, A. Z. Ghalam, T. Katsouleas, C. K. Huang, V. K. Decyk, C. Ren, W. B. Mori, F. Zimmermann and F. Ruggiero, PRST-AB 6, 081002 (2003).

[7] M. Pivi, Proc. PAC07, paper THPAS066.

[8] M. A. Furman, CBP Technote-387, 12 Nov. 2007.

[9] A. Chao, Physics of Collective Beam Instabilities in High Energy Accelerators, John Wiley \& Sons (2008).

[10] http://conf-ecloud02.web.cern.ch/confecloud02/CodeComparison/instres2008.html (maintained by F. Zimmermann).

\section{DISCLAIMER}

This document was prepared as an account of work sponsored by the United States Government. While this document is believed to contain correct information, neither the United States Government nor any agency thereof, nor The Regents of the University of California, nor any of their employees, makes any warranty, express or implied, or assumes any legal responsibility for the accuracy, completeness, or usefulness of any information, apparatus, product, or process disclosed, or represents that its use would not infringe privately owned rights. Reference herein to any specific commercial product, process, or service by its trade name, trademark, manufacturer, or otherwise, does not necessarily constitute or imply its endorsement, recommendation, or favoring by the United States Government or any agency thereof, or The Regents of the University of California. The views and opinions of authors expressed herein do not necessarily state or reflect those of the United States Government or any agency thereof, or The Regents of the University of California.

Ernest Orlando Lawrence Berkeley National Laboratory is an equal opportunity employer. 